\title{
New UV Absorbers for Cosmetic Sunscreens - A Breakthrough for the Photoprotection of Human Skin
}

\author{
Bernd Herzog*, Dietmar Hüglin, Elek Borsos, Albert Stehlin, and Helmut Luther \\ Sandmeyer Prize Laureates 2004
}

\begin{abstract}
Two new UV filters for use in cosmetic sunscreens have been developed. Bis-ethylhexyloxyphenol methoxyphenyl triazine (BEMT) is a hydroxy-phenyl-triazine derivative, which has been designed for optimal spectral performance, excellent photostability, and solubility in cosmetic oils. Methylene bis-benzotriazolyl tetramethylbutylphenol (MBBT) is the active ingredient of a particulate UV-filter system, which is available as a $50 \%$ dispersion of MBBT. Since the UV-attenuating efficacy depends strongly on particle size, the material is micronised to particle sizes below $200 \mathrm{~nm}$. The mode of action of this photostable filter system is governed to about $90 \%$ by absorption and $10 \%$ by scattering of UV light. Both filters show broad-spectrum characteristics with significant protection in the UVA range.
\end{abstract}

Keywords: Particle size · Photostability · Sunscreen · UV absorber · UVA · UVB

\section{Introduction}

In the 1930s UV absorbers were discovered as active ingredients of sun-protection formulations for human skin. Triggered by higher incomes and possibilities for travelling the sunscreen market started to develop in the 1950s and 1960s. In 1956, the sun protection factor (SPF), which enables the assessment of the performance of sunscreens, was invented by Schulze [1]. It is defined as the ratio of doses of solar radiation leading to reddening of human skin in presence and absence of the respective sunscreen. The broad introduction of the SPF on sunscreen packages in the 1970s revolutionized the market, since now the products became comparable on a quantitative basis. At the end of the 1970s the first products appeared that had achieved the 'magic' number of 20 , and in the 1980s the market

${ }^{\star}$ Correspondence: Dr. B. Herzog

Ciba Specialty Chemicals Inc.

G-9001.2.28

P.O. Box 1266

D-79630 Grenzach-Wyhlen

Tel.: +497624 122817

Fax: + 497624122888

E-Mail: bernd.herzog@cibasc.com further developed dynamically with diversity in SPF values as well as in product forms. Since the end of the 1980s the attitude of the consumers towards sun exposure has started to change. A brownish tan is still desirable, but people became increasingly aware of the harmful effects of the sun, above all the potential for skin cancer induction. With this background, the role of cosmetic sunscreens nowadays has changed from mere protection against sunburn to health prophylaxis.

Solar radiation in the spectral range between 290 and $400 \mathrm{~nm}$ is regarded as potentially dangerous for human skin, where the harmful effects strongly increase with smaller wavelength or higher energy of the radiation. The reason for not considering light of wavelengths below $290 \mathrm{~nm}$ is the filtering effect of the stratospheric ozone layer towards radiation of such kind. The spectral range between 400 and $320 \mathrm{~nm}$ is called UVA (400-340 nm: UVA I, 340-320 nm: UVA II), the range between 320 and $290 \mathrm{~nm}$ UVB, and light of wavelength below $290 \mathrm{~nm}$ is named UVC.

\subsection{UV Spectroscopic Requirements for UV Filters Used in Sunscreens}

First of all, sunscreens are expected to protect against sunburn, also called UV erythema. Fig. 1 shows the action spectrum of human skin for an erythemal reaction [2], the intensity spectrum of solar radiation at the surface of the earth [3], and the erythemal effectiveness, which is obtained from the convolution of erythemal action spectrum and the solar intensity spectrum. Thus, the erythemal effectiveness describes the relative spectral ability of solar UV radiation to induce erythema. It shows a maximum at $306 \mathrm{~nm}$, which explains why, until the early 1980s, in sunscreens only UVB filters were used.

Today, most sunscreens also contain UVA filters. There are several reasons:

- UVA contributes significantly to erythema formation, which can be estimated from the corresponding area under the erythemal effectiveness curve, and amounts to about $10-15 \%$ of the effect of the total UV. With pure UVB protection, $10-15 \%$ of the radiation would be transmitted without attenuation so that the total transmission of UV would always be higher than $10 \%$. Thus, the maximum SPF which can be achieved with a sunscreen based on pure UVB filters is about 10. In order to provide sunscreens with higher SPF it is necessary to add UVA absorbers.

- Animal experiments showed that the action spectrum for induction of skin cancers follows the erythemal action spectrum of human skin [4]. Assuming that the action spectrum for skin cancer induction is similar in humans, UVA has a certain contribution. 


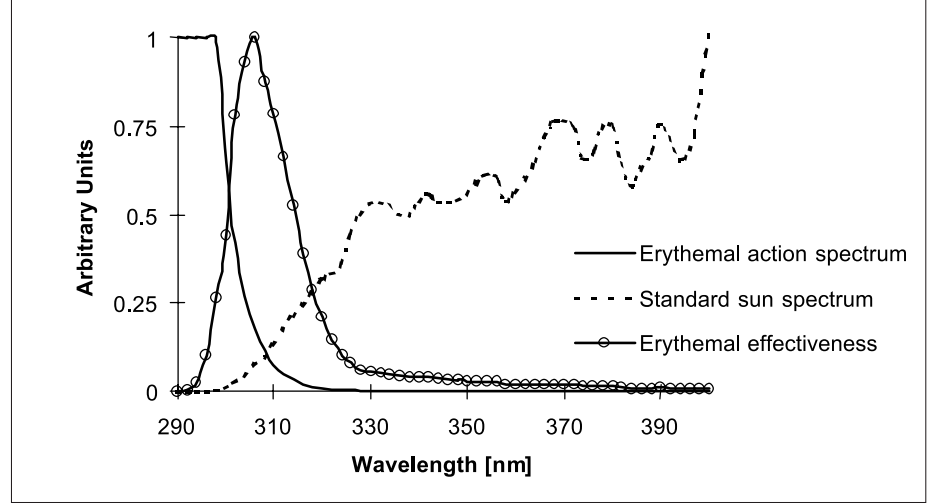

Fig. 1. Erythemal action spectrum, spectrum of standard sun and erythemal effectiveness

- UVA penetrates deeper into human skin than UVB and therefore damages the collagen structure. This explains the fact that UVA rather than UVB contributes to premature skin aging.

The UV spectra of the worldwide most employed UVB absorber (ethylhexyl methoxycinnamate, EHMC) and UVA absorber (butyl methoxy dibenzoylmethane, BMDBM) in ethanol are shown in Fig. 2. The $\mathrm{E}(1,1)$ is the extinction of $1 \%$ active (w/v) at $1 \mathrm{~cm}$ optical pathlength. Like EHMC and BMDBM, the majority of UV absorbers used in sunscreens are organic molecules with an UV chromophore. On the other hand, inorganic materials like $\mathrm{TiO}_{2}$ and $\mathrm{ZnO}$ play also a significant role in the market. They act via semiconductor absorption (with high band gap energy) in the solid state and therefore are applied as micro-fine particles, which show an additional scattering effect [5]. The extinction spectra of $\mathrm{TiO}_{2}$ and $\mathrm{ZnO}$ in aqueous dispersion are depicted in Fig. 3. $\mathrm{TiO}_{2}$ may be classified as a UVB filter, and $\mathrm{ZnO}$ is a weak broad-spectrum filter mostly used for UVA protection.

In order to achieve a balanced protection, in most sunscreens a blend of several UV filters is incorporated, which may consist of five or even more different individual filters.

\subsection{Solubility}

Most of the organic UV absorbers are oil- soluble or even oil-miscible compounds and consequently are incorporated into the oilphase of sunscreen emulsions. The typical concentration range of individual UV absorbers in sunscreen emulsions is between 1 and $5 \%$ or even more. Since the oil phase only makes up part of the emulsion, as a rule of thumb the solubility of the UV absorber in the pure oil has to be about three times higher than the desired concentration in the final formulation. Typical classes of oils used in cosmetic sunscreens are triacylglycerides, benzoic acid esters, carboxylic acid esters, dicarboxylic acid esters, pyrrolidones, and even UV absorbers like EHMC.

\subsection{Photostability}

Some of the UV absorbers commonly used are instable when irradiated with UV light. The most prominent example is BMDBM, which is reduced to less than $50 \%$ of its original concentration after $2 \mathrm{~h}$ of standard solar radiation [6]. There are other UV absorbers which act as photostabilisers for BMDBM. But the combination of BMDBM and EHMC results in increased photoinstability of both filters [7]. High photostability is a desirable property of new UV absorbers to be developed.

\subsection{Direction for the Development of New UV Absorbers}

At the time that the development of the new filters reported here began, the only generally available UVA filters were BMDBM and $\mathrm{ZnO}$, which both show certain shortcomings. As mentioned, the photostability of BMDBM is rather poor, and this problem becomes worse when BMDBM is combined with EHMC. For that reason BMDBM in most cases is not used together with this important UVB absorber. $\mathrm{ZnO}$, though photostable, has a poor efficacy in attenuating UVA. This situation suggested that there was a certain need for new efficient and photostable UVA filters.

\section{Bis-Ethylhexyloxyphenol Methoxyphenyl Triazine (Tinosorb ${ }^{\circledR} \mathrm{S}$ )}

Bis-ethylhexyloxyphenol methoxyphenyl triazine (BEMT), a hydroxy-phenyltriazine derivative, was developed as an oilsoluble, photostable, broad-spectrum UV absorber efficiently covering both the UVA and UVB range.

\subsection{UV Spectroscopic Properties}

Fig. 4 shows the dependence of the shape of the UV spectrum on the molecular structure for some hydroxy-phenyltriazines. The strong absorption of tri-phenyltriazines shown in the UVB range (see Fig. 4 , case A) has $\pi \pi^{*}$-character. An $n \pi *$-transition may also contribute to this band [8]. As an ortho-hydroxy group is introduced (Fig. 4, case B), a UVA band emerges, which is due to an intramolecular charge transfer $\left(\pi \pi^{*}-\mathrm{CT}\right)$. With two ortho-hydroxy groups at different phenyl-moieties this UVA absorption increases (Fig. 4, case D) and with three even more (Fig. 4, case C). The optimised broad-spectrum structure was obtained with case D (Fig. 4) referring to BEMT, which shows absorption maxima at 310 and $343 \mathrm{~nm}$ with $\varepsilon_{\max }=$ 46800 and $51900 \mathrm{M}^{-1} \mathrm{~cm}^{-1}$, respectively, measured in ethanol. Fig. 5 shows the $\mathrm{E}(1,1)$ spectrum and chemical structure of

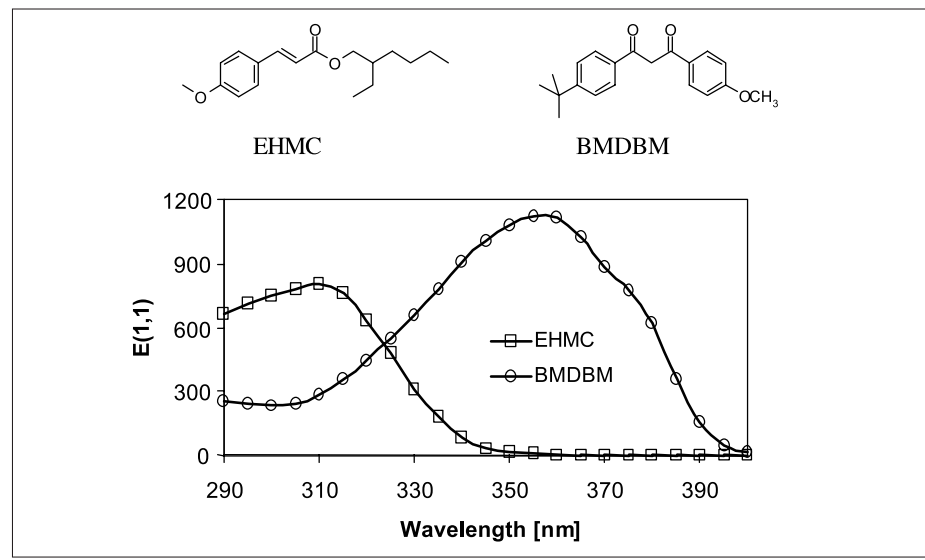

Fig. 2. UV spectra of ethylhexyl methoxycinnamate (EHMC; Parsol MCX, DSM) and butyl methoxy dibenzoylmethane (BMDBM; Parsol 1789, DSM)

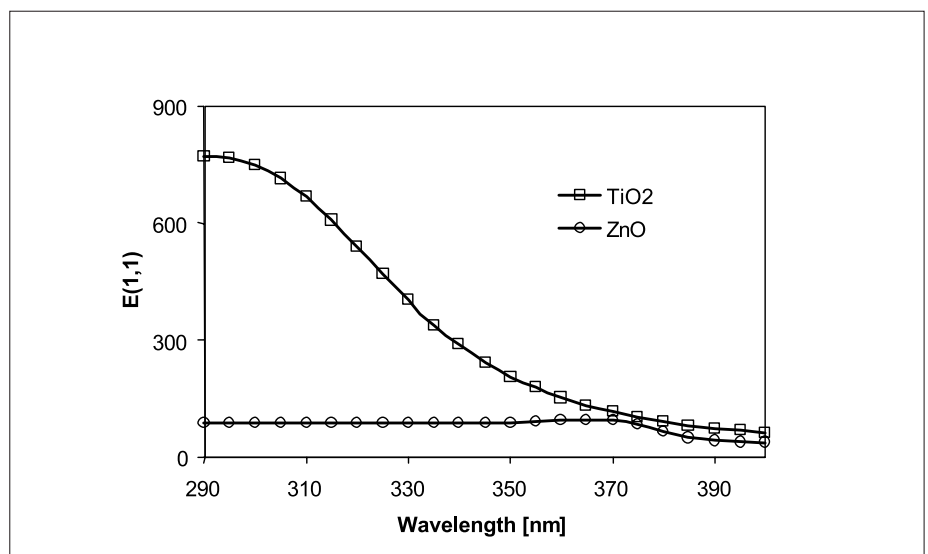

Fig. 3. UV spectra of $\mathrm{TiO}_{2}$ (Eusolex T2000, Merck) and ZnO (Symrise) 


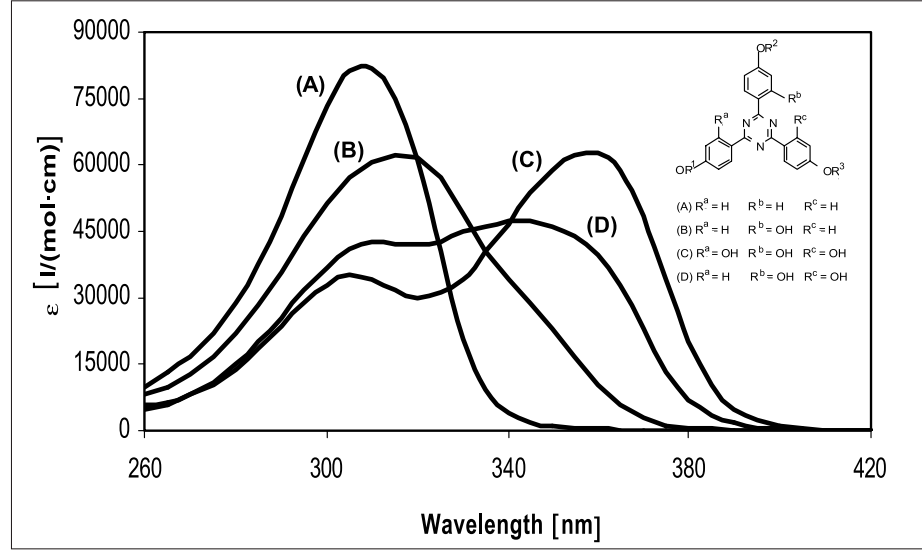

Fig. 4. UV spectral performance of hydroxy-phenyl-triazine derivatives as function of substitution; $R^{1}$ and $R^{3}=2$-ethyl-hexyl and $R^{2}=$ methyl

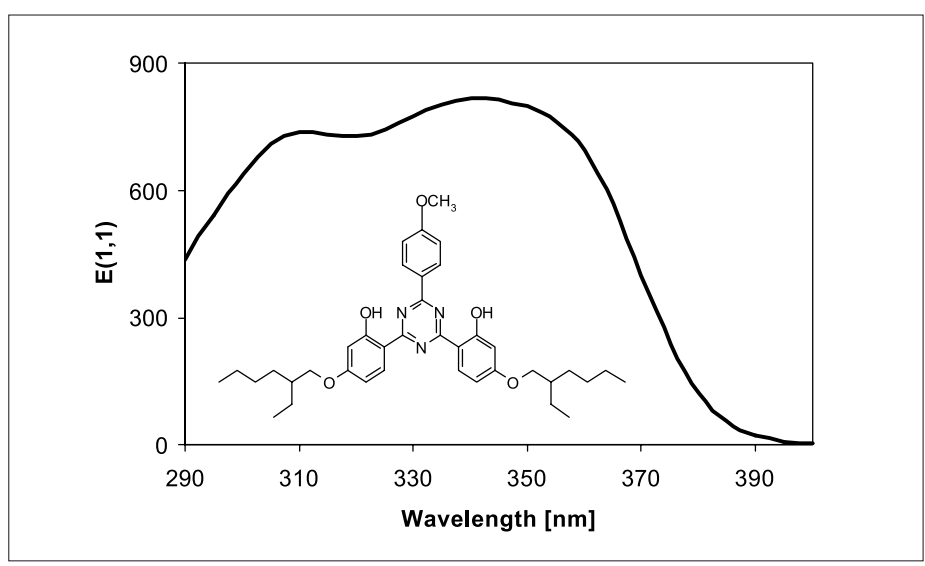

Fig. 5. UV spectrum and chemical structure of bis-ethylhexyloxyphenol methoxyphenyl triazine (BEMT); $\mathrm{E}(1,1)$ is the extinction at $1 \%$ concentration and $1 \mathrm{~cm}$ optical pathlength

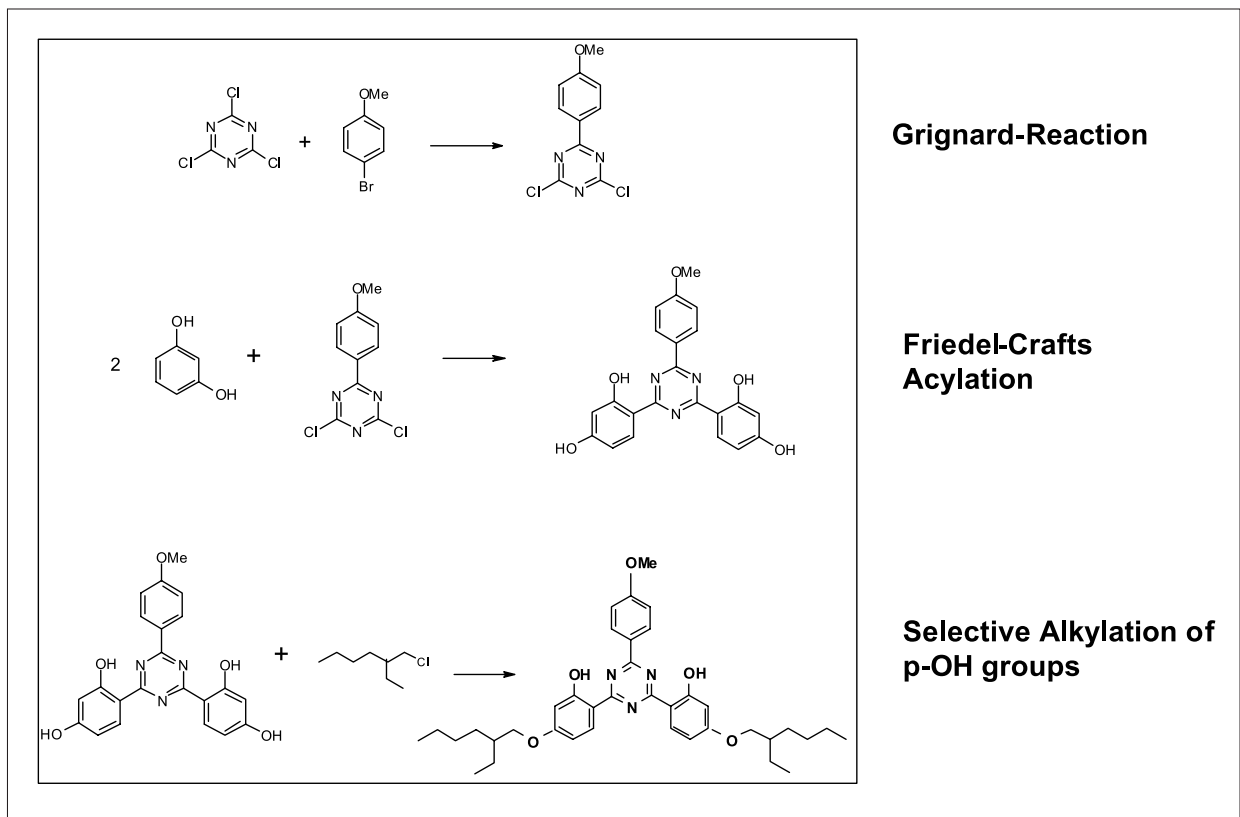

Fig. 6. Synthesis of bis-ethylhexyloxyphenol methoxyphenyl triazine (BEMT)

Table 1. Solubilities of BEMT and BMDBM in some frequently used cosmetic oils

\begin{tabular}{lcc} 
& BEMT & BMDBM \\
\hline $\mathrm{C}_{12}-\mathrm{C}_{15}$ Alkyl Benzoate & $13 \%$ & $11 \%$ \\
Isopropy Myristate & $6 \%$ & $9 \%$ \\
Caprylic/Capric Triglyceride & $5 \%$ & $7 \%$ \\
Propyleneglycol Dicaprate/Dicaprylate & $7 \%$ & $5 \%$ \\
Dimethicone & $<1 \%$ & $<1 \%$
\end{tabular}

\section{BEMT.}

\subsection{Synthesis}

The synthesis of BEMT is a three-step process, which is shown schematically in Fig. 6. After further purification steps the final product is obtained with a purity of $>98 \%$.

\subsection{Solubility of BEMT}

The ethylhexyl residues in the BEMT molecule are introduced to increase the sol- ubility in cosmetic oils (Fig. 5). The solubility in cosmetic solvents is a key parameter of UV absorbers. Solubility was determined by stirring an excess of the substance for seven days at $25^{\circ} \mathrm{C}$. After removal of the remaining solid by centrifugation and filtration, the dissolved amount was determined by UV spectroscopy. In Table 1 solubility data of BEMT and BMDBM are compared for a number of frequently used oils. The solubilities of both UV absorbers are comparable in most solvents.

\subsection{Photostability of BEMT}

Excitation of the molecule by a photon increases the energy of the molecule, which switches from the electronic $S_{0}$ ground state to the first electronic excited state $S_{1}$. After excitation different processes are possible like fluorescence, intersystem crossing, phosphorescence, internal conversion (IC), or photoreactions. When internal conversion (IC) is the fastest and therefore dominating pathway, a redistribution of the absorbed energy from electronic to vibrational excitation takes place. In contrast to the electronically excited molecule, the vibrationally excited one can be deactivated by collisions with surrounding molecules, dissipating the energy into harmless heat. The faster the rate of internal conversion, the higher will be the photostability [9]. The energy-gap law [10] states that the rate of internal conversion becomes faster as the energy gap between ground state and excited state decreases. This for instance is the case with molecules that can undergo an excited state proton transfer (also called photo-tautomerism).

BEMT contains two intramolecular hydrogen bridges (between the ortho-OH groups and the triazine nitrogen atoms) that enable an excited-state intramolecular proton transfer after photoexcitation. Excitation goes from the electronic ground state $\mathrm{S}_{0}$ to the first electronically excited state $\mathrm{S}_{1}$ (Fig. 7). The state $S_{1}$, , which is reached after isomerization, has less energy than $S_{1}$. After internal conversion the molecule relaxes from $\mathrm{S}_{0}{ }^{*} \rightarrow \mathrm{S}_{0}$, . Since the ground state $\mathrm{S}_{0}$ ' of the isomer is energetically higher than the ground state $S_{0}$, the deactivated isomer reacts back immediately to the original ground state $S_{0}$. The whole proton transfer cycle lasts less than one picosecond $\left(10^{-12} \mathrm{~s}\right)$. Since it is much faster than the other processes which may occur after excitation, it is a very efficient deactivation mechanism. Thus, the presence of orthohydroxy groups not only positively influences the shape of the absorption spectrum, but also the photostability. 


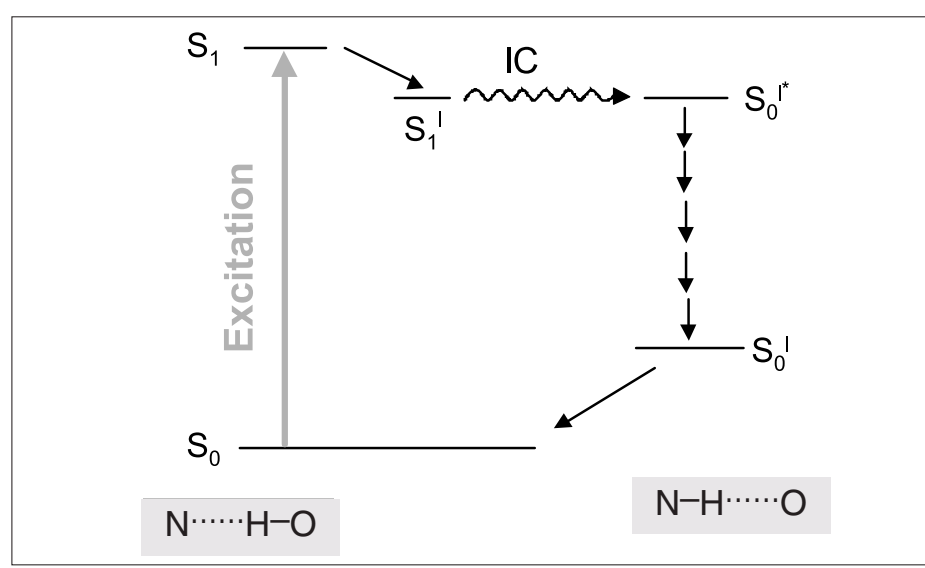

Fig. 7. Dissipation of absorbed energy via the photo-tautomerism cycle

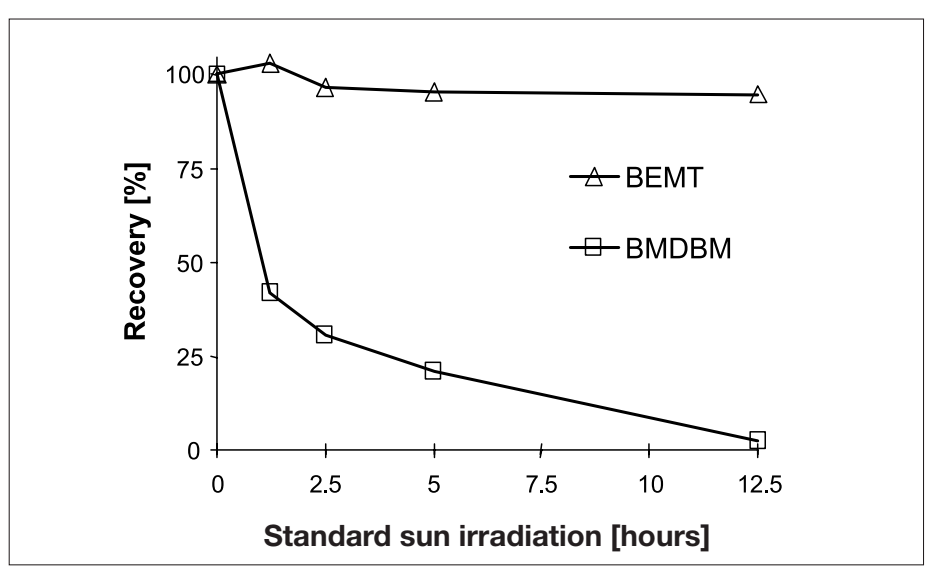

Fig. 8. Recovery of the parent molecules after simulated solar irradiation
In Fig. 8 the photostability of BEMT is compared to that of BMDBM. For the experiment emulsions containing one of the filters mentioned were distributed on quartz plates with rough surface to an amount of $2 \mu \mathrm{l} / \mathrm{cm}^{2}$. Realistic concentrations were chosen and adjusted to achieve the same average extinctions in the UV range $(290-400 \mathrm{~nm})$ of about 2.2. The samples were irradiated in a solar simulator (Atlas, CPS plus), the spectrum of which corresponded to that of standard sunlight [3] referring to conditions at Albuquerque (New Mexico, latitude $35^{\circ} \mathrm{N}$ ). Exposure for $15 \mathrm{~min}$ to this type of irradiation leads to erythema with persons of skin type II (Caucasians) [11]. Thus, the longest irradiation of $12.5 \mathrm{~h}$ in the experiment shown in Fig. 8 is the 50fold of this time, and in order to avoid sunburn when exposed to this UV dose, a sunscreen with an SPF of 50 would be necessary. After irradiation, the samples were analysed by HPLC quantifying the recovery of the parent molecule. Within the range of experimental error BEMT remains stable up to the highest UV dose, whereas BMDBM is degraded [12].

\section{Methylene Bis-Benzotriazolyl Tetramethylbutylphenol (Tinosorb $\left.{ }^{\circledR} M\right)$}

Methylene bis-benzotriazolyl tetramethylbutylphenol (MBBT) is the active ingredient of Tinosorb ${ }^{\circledR} \mathrm{M}$, which is an aqueous dispersion with the composition shown in Table 2. The structure of MBBT is depicted in Fig. 9.

As already mentioned, sufficient solubility in cosmetic oils (or in water) is a crucial property of UV filters intended for use in sunscreens. With certain chromophores of excellent UV-absorbing properties, it is hardly possible to obtain acceptable solubility. To make a virtue of necessity, in such cases one may strive for weakest solubility in order to create organic UV absorbers as microfine particles as already known from the inorganic filters $\mathrm{TiO}_{2}$ and $\mathrm{ZnO}$, and

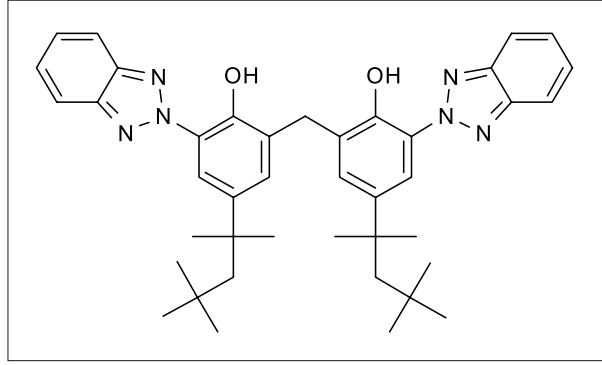

Fig. 9. Chemical structure of methylene bisbenzotriazolyl tetramethylbutylphenol (MBBT)

Table 2. Composition of Tinosorb ${ }^{\circledR} M$

$\begin{array}{ll}\text { Organic micropigment (MBBT) } & 50 \% \\ \text { Surfactant (decyl glucoside) } & 7.5 \% \\ \text { Thickener (xanthan gum) } & 0.2 \% \\ \text { Propylene glycol } & 0.4 \% \\ \text { Water } & \text { ad } 100 \%\end{array}$

from coloured organic pigments. This concept was realised for the first time with Tinosorb ${ }^{\circledR} \mathrm{M}$.

\subsection{Micronisation of MBBT}

The solubility of MBBT in water is extremely low $\left(<10^{-6 \%}\right)$ and in most cosmetic oils it is $<0.2 \%$. Therefore the substance is well suited for micronisation, which is achieved with a milling process in deionised water using decyl glucoside as dispersant [13]. After pre-milling with a corundum disc mill, the main milling procedure is performed with a ball-mill. Xanthan gum as a thickener is added after the milling is finished in order to avoid sedimentation of particles. The composition of the final dispersion is shown in Table 2.

In order to assess particle sizes in a range of about 10 micrometers to less than 50 nanometers, appropriate particle size analytics had to be set up. Fig. 10 shows the cumulative volume distribution of particles of the final product measured with fibre-optic quasi-elastic light scattering (FOQELS) [14]. The so-called median or $\mathrm{d}(0.5)$-value of the polydisperse cumulative volume size distribution is defined as the diameter up to which the area under the distribution curve is equal to $50 \%$ of the whole area. The $\mathrm{d}(0.5)$ value of the final dispersion is between 150 and $170 \mathrm{~nm}$.

Fig. 11 shows the progress of the ballmilling process in terms of the $d(0.5)$-value as function of the energy consumption of the mill. Most of the energy is needed to achieve further particle size reduction in the sub-micrometer range.

\subsection{UV Spectroscopic Properties of MBBT}

Fig. 12 shows the UV spectrum of particulate MBBT with $\mathrm{d}(0.5)=169 \mathrm{~nm}$ and the UV spectrum of MBBT dissolved in dioxane. The absorption spectrum of the UV absorber in solution shows a double band structure. The longer wavelength band occurring at $350 \mathrm{~nm}$ can be attributed to a $\pi \pi *$-charge transfer (CT) state. This is favoured by the planar orientation enforced by the intramolecular hydrogen bond, made possible by the hydroxy group in ortho-position. The shorter wavelength band at about $305 \mathrm{~nm}$ arises from a local transition within the benzotriazole moiety [15]. As in the case of BEMT, the intramolecular hydrogen bond enables also in MBBT an excited-state intramolecular proton transfer after photoexcitation resulting in excellent photostability of the molecule [7].

The spectrum of particulate MBBT shows distinctive differences to that in solution, which are not explained by the additional scattering effect: The longer wavelength band is shifted to $360 \mathrm{~nm}$ and is followed by a shoulder at $380 \mathrm{~nm}$. The short wavelength band at $305 \mathrm{~nm}$ shows no significant shift, but there is also an additional shoulder at $320 \mathrm{~nm}$. These spectral changes are attributed to electronic interactions of 


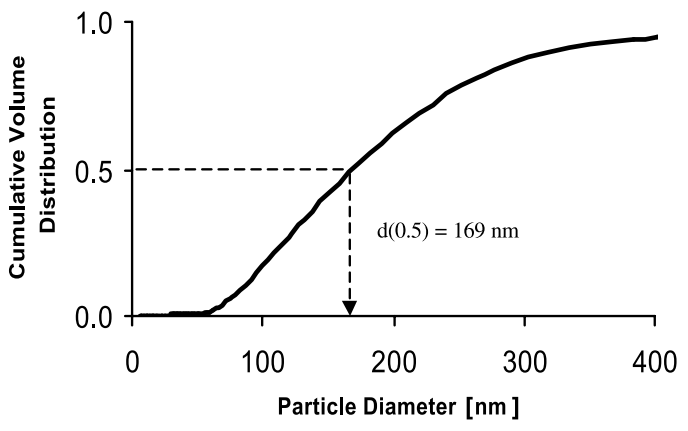

Fig. 10. Cumulative particle size distribution of MBBT after the milling process

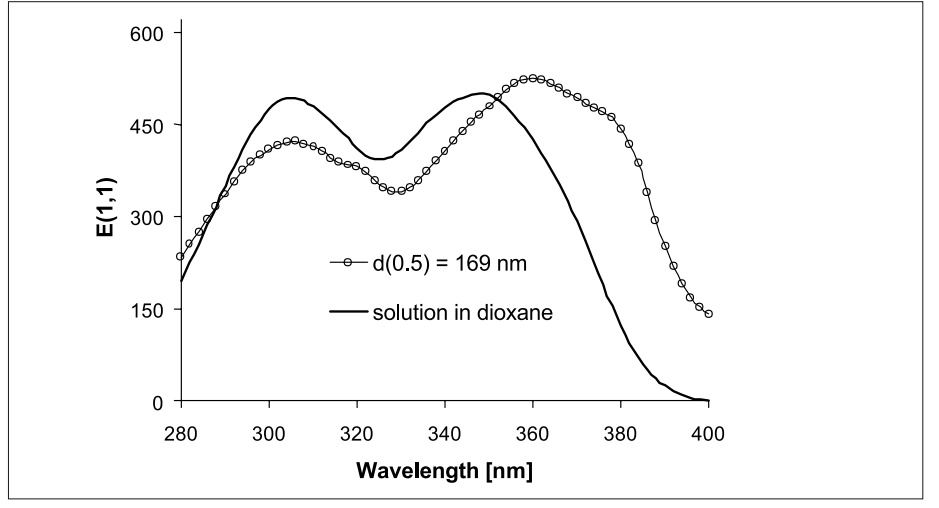

Fig. 12. UV spectra of particulate MBBT with particle size $d(0.5)=$ $169 \mathrm{~nm}$ and MBBT dissolved in dioxane

the chromophores in the particles [16] and depend on the particle size [17].

\subsection{Dependence of UV Attenuation on Particle Size}

For organic coloured pigments the absorption increases with smaller particles while the scattering shows a maximum at a certain particle size [18]. The extinction results as a superposition of both effects, and thus may show an optimum at a certain particle size.

To measure the UV attenuating efficiency of the MBBT particles dispersions were prepared in a concentration range between 0.5 and $3 \%(\mathrm{w} / \mathrm{v})$ and filled into sandwichtype quartz cells of $0.0008 \mathrm{~cm}$ thickness. For measurements an UV/vis spectrometer with integration sphere accessory was used in order to collect also the light scattered in forward direction by the turbid samples. The set-up shown in Fig. 13a was employed for this purpose. In the concentration range applied, Lambert-Beer's law functionality was obeyed. The results in Fig. 14 show a strong effect of the particle size on the extinction. The smaller the particles become, the more efficient they are. One reason is that in larger particles the UV-absorbing molecules in the interior are shielded by the outer ones. Thus, a part of the molecules will not be effective in absorbing UV radiation. Furthermore, with smaller particles the UV-absorbing material is more evenly distributed in the volume than with larger ones.
In Fig. 14 the highest extinction is achieved at a particle size of $\mathrm{d}(0.5)=0.16$ $\mu \mathrm{m}$, which corresponds to the end point of the milling process. The question arises whether further micronisation would lead to even higher extinction. In order to achieve smaller particle sizes, the dispersion resulting at the end of the milling process was fractionated by centrifugation. Depending on centrifugation time and speed a certain portion of the larger particles is found in the sediment, whereas the smaller ones remain in the supernatant, and thus can be isolated. Fig. 15 shows the extinction $\mathrm{E}(1,1)$ at 360 $\mathrm{nm}$ as function of particle sizes achieved during milling and centrifugation [17]. There is an optimum particle size at $\mathrm{d}(0.5)=80 \mathrm{~nm}$, and with particles smaller than $80 \mathrm{~nm}$, the extinction decreases again. Obviously, at this point the absorption has reached a saturation level, but further micronisation causes less scattering resulting in lower extinction. In terms of the $\mathrm{E}(1,1)$ at $360 \mathrm{~nm}$, the MBBT particles at the end of the milling process show about $90 \%$ of the highest possible performance.

\subsection{Quantification of the Ratio of Absorption and Scattering}

From reflectance measurements the coefficients of absorption, $\mathrm{K}$, and scattering, $\mathrm{S}$, can be evaluated [19]. An UV/vis spectrometer with integration sphere accessory was

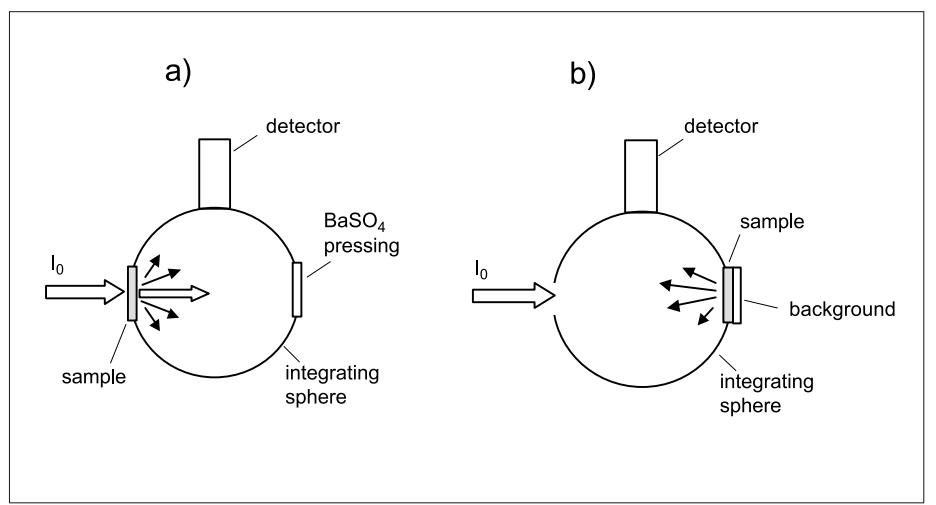

Fig. 13. Set-up of UV/vis spectroscopic experiments with integration sphere: a) measurement of diffuse transmission, b) measurement of diffuse reflectance

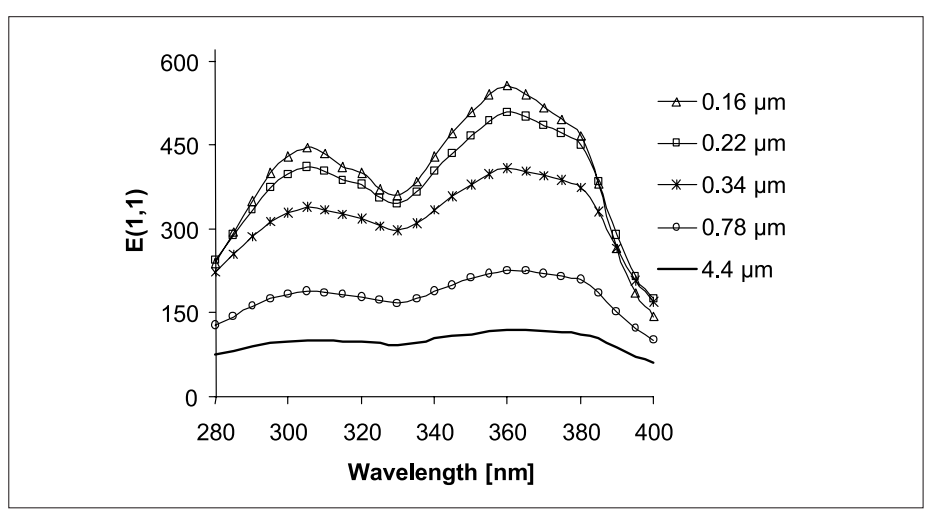

Fig. 14. UV spectra of MBBT dispersions at different particle sizes 


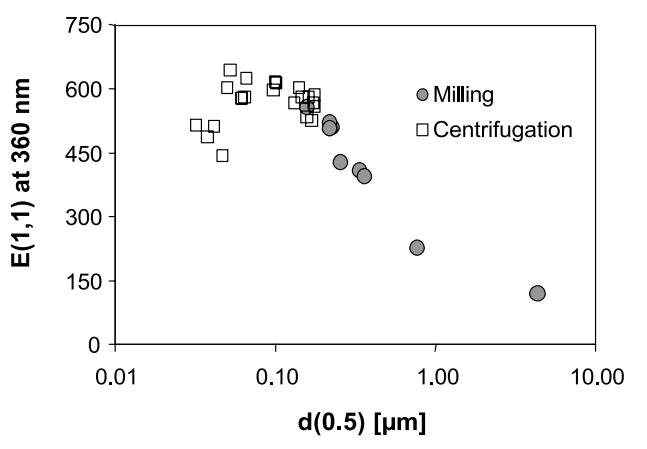

Fig. 15. Extinctions $E(1,1)$ at $360 \mathrm{~nm}$ of MBBT dispersions of a range of particle sizes between $40 \mathrm{~nm}$ and $4 \mu \mathrm{m}$

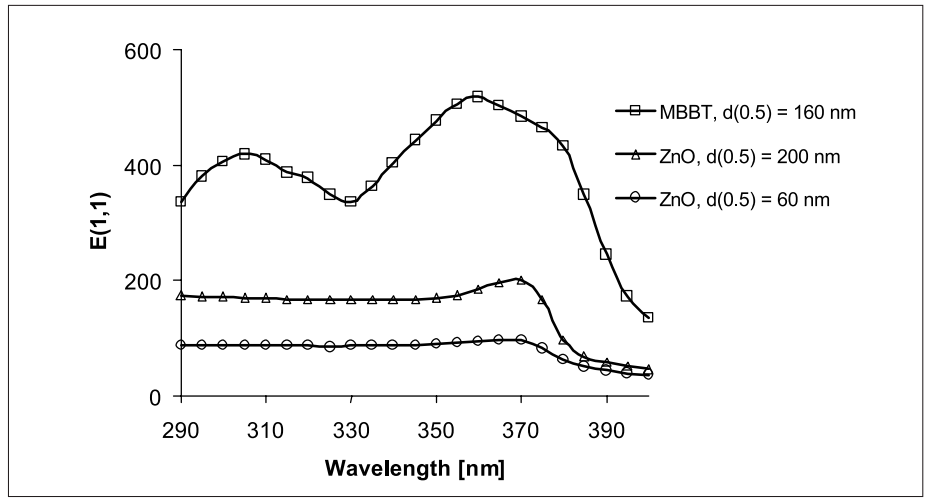

Fig. 17. Comparison of extinction $\mathrm{E}(1,1)$ of particulate MBBT and $\mathrm{ZnO}$ at two different particle sizes

used for this purpose with the set-up shown in Fig. 13b. Diluted suspensions (1-3\%) of the particulate UV absorber obtained after the milling process $(\mathrm{d}(0.5)=0.16 \mu \mathrm{m})$ were filled into quartz cells with $0.0008 \mathrm{~cm}$ optical thickness and mounted in the sample holder at the corresponding window of the sphere. The reflection of the samples was measured on white and on black background. With white background the signal measured in the sphere corresponds to the light transmitted and scattered by the sample. Since the black background absorbs the light which is transmitted by the sample, the signal in that case is only due to scattered light. The results for $\mathrm{K}$ and S (Fig. 16) show that this particulate UV filter works mainly by absorption. Scattering contributes only to about $10 \%$ of the total effect.

\subsection{Comparison of MBBT and ZnO}

$\mathrm{ZnO}$ is a UV filter which is in some aspects comparable to MBBT: Both are particulate filters and both are used for UVA protection, having broad-spectrum characteristics at the same time. Fig. 17 shows the $\mathrm{E}(1,1)$ spectra of $\mathrm{ZnO}$ with two different particle sizes and MBBT. ZnO with smaller particle size is more efficient than that with larger particles, but the extinction is still limited, and high concentrations are necessary for satisfactory UVA protection. Since the $\mathrm{E}(1,1)$ of MBBT is significantly higher, for the same effect less material has to be incorporated into sunscreen formulations.

\section{Concluding Remarks}

The development of Tinosorb ${ }^{\circledR} \mathrm{S}$ (BEMT) and Tinosorb ${ }^{\circledR} \mathrm{M}$ (MBBT) was possible by combining synthesis research, dispersion technology and physicochemical expertise. It was initiated by realizing certain market needs, which emerged by a new understanding of what is necessary for the protection of human skin against UV radiation. Today these products are present in many sunscreens of different producers. The most prominent features of both filters are the efficient coverage of the UVA range but showing broad-spectrum characteristics at the same time, and their photostability.

Meanwhile the situation on the sunscreen market has changed, since also other producers of UV filters have developed new UVA absorbers [20]. A crucial question is whether there is still space for further developments. Regarding the dynamics of the sunscreen market during the past decades, we strongly believe that the answer will be positive.

Received: June 14, 2004

[1] R. Schulze, Parfüm. Kosmet. 1956, 37, 310; R. Schulze; Parfüm. Kosmet. 1956, 37, 365.

[2] A.F. McKinlay, B.L. Diffey, CIE Journal 1987, 6, 17.

[3] B.L. Diffey, J. Robson, J. Soc. Cosm. Chem. 1989, 40, 127.

[4] F.R. de Gruijl, H.J.C.M. Sterenborg, P.D. Forbes, R.E. Davies, C. Cole, G. Kelfkens,
H. van Weelden, H. Slaper, J.C. van der Leun, Cancer Res. 1993, 53, 53.

[5] J.L. Robb, L.A. Simpson, D.F. Tunstall, Drug Cosmetic Industry 1994, 154, 32.

[6] G. Berset, H. Gonzenbach, R. Christ, R. Martin, A. Deflandre, R.E. Mascotto, J.D.R. Jolley, W. Lowell, R. Pelzer, T. Stiehm, Int. J. Cosmet. Sci. 1996, 18, 167.

[7] B. Herzog, K. Sommer, Proc XXI ${ }^{\text {st }}$ IFSCC Int. Congr. 2000, P60.

[8] G.J. Stueber, M . Kieninger, H . Schettler, W. Busch, B. Goeller, J. Franke, H.E.A. Kramer, H. Hoier, S . Henkel, P. Fischer, H. Port, G. Rytz, J.L. Birbaum, J. Phys. Chem. 1995, 99, 10097.

[9] J.E. Otterstedt, J. Phys. Chem. 1973, 58, 5716.

[10] J.A. Baltrop, J.D. Coyle, 'Excited states in organic chemistry', Chapter 3, London 1975, Wiley \& Sons Ltd., London, 1975.

[11] T.B. Fitzpatrick, Archives of Dermatology 1988, 124, 869.

[12] T. Rudolph, 'Photoreaktionen chemischer UV-Filter - Ein Beitrag zur Bewertung kosmetischer Lichtschutzmittel', Verlag Mainz, Aachen, 1996.

[13] a) W. Dümler, P. Fankhauser, H. Luther, WO 95 22959, 23 February 1994; b) H. Luther, A. Stehlin, M. Minklei, WO 97 03643, 6 February 1997.

[14] a) H. Wiese, D. Horn, J. Chem Phys. 1991, 97, 6429; b) B. Herzog, A. Katzenstein, K. Quass, A. Stehlin, H. Luther, J. Colloid Interface Sci. 2004, 271, 136.

[15] J. Rieker, E. Lemmert-Schmidt, G. Goeller, M. Roessler, G.J. Stueber, H. Schettler, H.E.A. Kramer, J.J. Stezowski, H. Hoier, S. Henkel, A. Schmidt, H. Port, M. Wiechmann, J. Rody, G. Rytz, M. Slongo, J.L. Birbaum, J. Phys. Chem. 1992, 96, 10225.

[16] J. Rieger, D. Horn, Angew. Chem. 2001, 113,446

[17] B. Herzog, K. Quass, E. Schmidt, S. Müller, H. Luther, J. Colloid Interface Sci. 2004, in press.

[18] D.H. Clewell Jr., J. Opt. Soc. Am. 1941, 31, 521.

[19] G. Kortüm, 'Reflexionsspektroskopie', Springer, Berlin, 1969.

[20] U. Osterwalder, H. Luther, B. Herzog, Bundesgesundheitsblatt 2001, 44, 463. 